\title{
Effects of Performance Feedback and Feedback Withdrawal on Auditory Looming Perception
}

\author{
Lawrence D. Rosenblum, Michael S. Gordon, and \\ A. Paige Wuestefeld \\ Department of Psychology \\ University of California-Riverside
}

\begin{abstract}
This research examined accuracy in an auditory time-to-arrival task when performance feedback was first provided to listeners and was subsequently withdrawn. Listeners made judgments about the time to arrival of an approaching car based on various portions of the event. Listeners participated in 3 experimental sessions on consecutive days. The experimental group received no feedback during the first session, feedback during the second session, and no feedback during the final session. When feedback was withdrawn, the higher performance level attained during training was retained. We discuss results in terms of theories of time-to-arrival perception and the importance of stimulus naturalness.
\end{abstract}

Researchers have investigated how listeners make anticipatory judgments of time to arrival of an approaching sound source (Hellman, 1993; Rosenblum, Wuestefeld, \& Saldaña, 1993; Schiff \& Oldak, 1990). Typically in these experiments, listeners are presented with a looming sound source signal that vanishes en route and are asked to judge when the source would have reached them had it continued at the same rate. The time between when the signal ends and when the source would ostensibly reach the listener, the occluded period, can vary in length. In one study, Schiff and Oldak found that for most occluded periods, sighted humans were not particularly accurate at judging auditory time to arrival and that auditory information did not enhance visual judgments of time to arrival. However, they did find that blind listeners' auditory time-to-arrival judgments were comparable to that of sighted perceivers making

Requests for reprints should be sent to Lawrence D. Rosenblum, Department of Psychology, University of California-Riverside, Riverside, CA 92521.E-mail: rosenblu@citrus.ucr.edu 
analogous visual judgments. This finding suggested that experience can play a role in auditory time-to-arrival perception.

In another study, Rosenblum et al. (1993) found that although anticipatory performance of sighted listeners was initially mediocre, judgments improved significantly when listeners were provided with performance feedback. In that study, listeners were presented with monaural recordings of a looming car. On each trial, they were given feedback in the form of a time line specifying how early or late their judgment was. Rosenblum et al. observed that the performance of participants who received feedback was significantly better than that of participants who received no feedback. Thus, practice or training can be an important component for accurate auditory time-to-arrival perception. Because sighted listeners are primarily visually guided, it may be that the accuracy in auditory looming judgments requires experience through either implicit or explicit feedback. Although the results of the Rosenblum et al. study underscore the importance of learning, it is unclear exactly how that learning occurs. We designed this study to examine this question, using a learning-through-feedback paradigm.

\section{EFFECTS OF FEEDBACK ON LOOMING PERCEPTION}

Jagacinski, Johnson, and Miller (1983) tested the immediate and sustained influence of judgment feedback on visual looming perception. Participants were presented with a vertical target line moving horizontally right to left and then reversing direction. After participants became familiar with the trajectory, experimental trials began in which the line disappeared midway through the event, at which point a prediction line appeared. Participants were asked to anticipate the arrival of the visual target by indicating with a button press when the target line would have reached the prediction line. In the absence of feedback, performance was mediocre: Participants seemingly added a large accelerative component to their extrapolated trajectories. However, when participants were given performance feedback (in seconds) that indicated their judgment error, their performance improved substantially. Jagacinski et al. attributed this improvement to the "tuning" of internal model parameters. When participants returned on the following day, feedback was withdrawn, and performance reverted back to its original, prefeedback level. The authors reasoned that at least one of the parameter values of the internal model was not retained but instead shifted back to its initial value.

However, in more natural contexts, there are clear instances in which observers maintain improved approach judgments long after explicit feedback is withdrawn. This is certainly the case when children fine-tune anticipatory skills such as baseball batting and catching (e.g., DeLucia \& Cochran,1985; Whiting, Gill, \& Stephenson, 1970). With regard to auditory looming, Schiff and Oldak's (1990) research showed that blind listeners are substantially better than sighted 
listeners in judging looming sound sources. It can be assumed that at least one way blind individuals fine-tune this skill is through feedback. Although self-obtained feedback would be far too dangerous for calibrating judgments of oncoming vehicles, blind individuals might improve this ability through the verbal feedback provided by mobility trainers. This skill could also be fine-tuned by transferring experience from less threatening contexts in which obtaining feedback (e.g., haptic) would be feasible. Regardless, Schiff and Oldak's research showed that this improved performance is maintained long after explicit response feedback is available.

Thus, although the one looming study that specifically examined the influence of feedback withdrawal (Jagacinski et al., 1983) showed a reversion of performance, many anecdotal examples show long-term learning effects. Clearly, the experience accorded to the expert looming perceiver is much more extensive and more functionally relevant than the short-term feedback experience provided to the Jagacinski et al. participants. These facts could account for the differential results. However, it could be that the performance reversion observed in the Jagacinski et al. study was also related to the unnaturalness of their stimuli. The stimuli of their experiment were graphically presented and were not effected by any of the natural physical (e.g., inertial, frictional) constraints that normally act on looming objects. In contrast, the looming information provided to expert anticipators is generated in a context of natural physical constraints. For example, the auditory stimuli of Schiff and Oldak (1990) were naturally recorded; thus they were generated with the appropriate physical constraints. Perhaps, the long-term benefits of perceptual experience might be contingent on the naturalness of the available stimuli. The notion that stimulus naturalness plays a role in perceptual performance is a central tenet of ecological psychology (e.g., J. J. Gibson, 1966, 1979/1986; see also, Flynn, 1994; Runeson \& Vedeler, 1993) and also of other theoretical approaches (e.g., Brunswik, 1956), and it provided one of the motivating issues for the Schiff and Oldak experiments. This notion was also reflected in the theorizing of E. J. Gibson (1969) in her distinction between embellishment (cognitive) and differentiation (ecological) theories of perceptual learning.

In the following experiment, we implemented our looming-car methodology (Rosenblum et al., 1993) to test the influence of feedback withdrawal on auditory looming perception. The purpose of our study was twofold. First, we were interested in how feedback and withdrawal of feedback influences auditory looming judgments. The second, more general purpose of this study was to examine whether the withdrawal of feedback would have an influence on anticipatory judgments of more natural stimuli. We believe that our looming-car methodology (Rosenblum et al., 1993) provides a more natural stimulus context and, therefore, offers a good test of this question. The critical test in this experiment involved monitoring participant performance in sessions before, during, and after feedback was provided. 


\section{METHOD}

\section{Participants}

Thirty undergraduate students (10 men and 20 women) participated in this study. All participants received a combination of academic credit and monetary compensation. Each listener reported good hearing and vision when signing up for the experiment.

\section{Stimuli}

The stimuli used for this experiment were the same as those used by Rosenblum et al. (1993). Recordings of a car approaching on a bypass route were made in the free field with a unidirectional microphone (Sennheiser MKH 60 RF Condenser) and a high-quality monaural cassette recorder (Marantz No. PMD221). The recordings were made from the edge of an unpaved road so that the car passed about $3 \mathrm{ft}(0.9$ $\mathrm{m})$ from it, and the microphone was angled so that it was nearly parallel with the road and faced the oncoming vehicle. The angle formed by the microphone's direction and road was about $6^{\circ}$. Recordings were made with the car having a constant approach velocity of both 15 and $25 \mathrm{mph}$. During the $15-\mathrm{mph}$ approach, the car traveled about $140 \mathrm{ft}$, and during the $25-\mathrm{mph}$ approach, it traveled about $200 \mathrm{ft}$. Twenty recordings were made, and the best recordings for each approach velocity were chosen based on overall quality. Each of these two recordings were then sampled using a Compaq 386 computer with a sampling rate of $10 \mathrm{kHz}$ (8 bit). The recordings were low-pass filtered at $5 \mathrm{kHz}$.

Each of the sampled signals included the entire acoustic event. In other words, the signal did not stop at the point in which the car reached the microphone but also included a portion of the signal that came after passing the microphone (the time of arrival). The signal duration was $6,328 \mathrm{msec}$ and $5,578 \mathrm{msec}$ for the 15 - and 25 -mph signals, respectively. Each signal was then divided into three parts equal in duration. This resulted in three 2,109 msec thirds for the 15 -mph signal and three $1,859 \mathrm{msec}$ thirds for the 25 -mph signal. Dividing each signal into three equal segments allowed us to deliver different portions of the event to participants and test the efficacy of time-to-arrival information for different portions of the signal (and different occluded periods), and also test signals of different durations. A similar strategy for dividing the trajectory of a looming event was used in studying visual time-to-contact information (DeLucia \& Cochran, 1985).

We measured the point at which the car reached the microphone, the time of passage, for each signal by determining the time at which the intensity of the signal reached its peak, and then we verified this point by measurements taken during stimulus recording. For the $15-\mathrm{mph}$ signal, the time of passage occurred $714 \mathrm{msec}$ from the onset of the third portion, whereas for the 25 -mph signal it was $709 \mathrm{msec}$ from the onset of the third portion of the signal. 
These edited stimuli acted as the basic building blocks for stimulus trials. Dividing the stimuli into thirds allowed us to present seven different event types per speed condition to listeners. These different types are depicted graphically in Figure 1 . Types $\mathrm{A}, \mathrm{B}$, and $\mathrm{C}$ each contained the first, second, and third portion of the signal, respectively. Types $\mathrm{A}-\mathrm{B}, \mathrm{B}-\mathrm{C}$, and $\mathrm{A}-\mathrm{B}$ each contained a two thirds portion of the signal. Type A-B involved the first two thirds, Type B-C contained the last two thirds, and Type A-C contained the first and last third with the middle replaced by silence. Finally, Type A-B-C included the entire signal.

The stimuli were presented to participants directly from the computer over high quality headphones. The peak stimulus intensity of the headphones was $74 \mathrm{~dB}$ sound-pressure level (SPL) for the $15 \mathrm{mph}$ stimuli and $75 \mathrm{~dB}$ SPL for the $25 \mathrm{mph}$ stimuli.

\section{Procedure}

All participants participated in three 1-hr sessions held on consecutive days (Jagacinski et al., 1983). Each participant was assigned randomly to an experimental group. There were three experimental groups, each with 10 participants. The specific group assignment dictated whether the participant received judgment feedback during specific sessions in the experiment. For those participants who did receive feedback, feedback was given in the form of a time line (Rosenblum et al., 1993). After each judgment, a long horizontal bar was displayed, and the listener's accuracy was represented by a medium-sized vertical line drawn on the bar. The bar was labeled Early on the left end and Late on the right and had a small vertical line drawn in the middle indicating a perfectly correct response. Participants were told that the medium-sized mark indicated the degree to which their judgment was off relative to the actual time of passage. This type of feedback was used because it provided listeners with the direction as well as the degree to which their judgment was off.

Type A-B-C

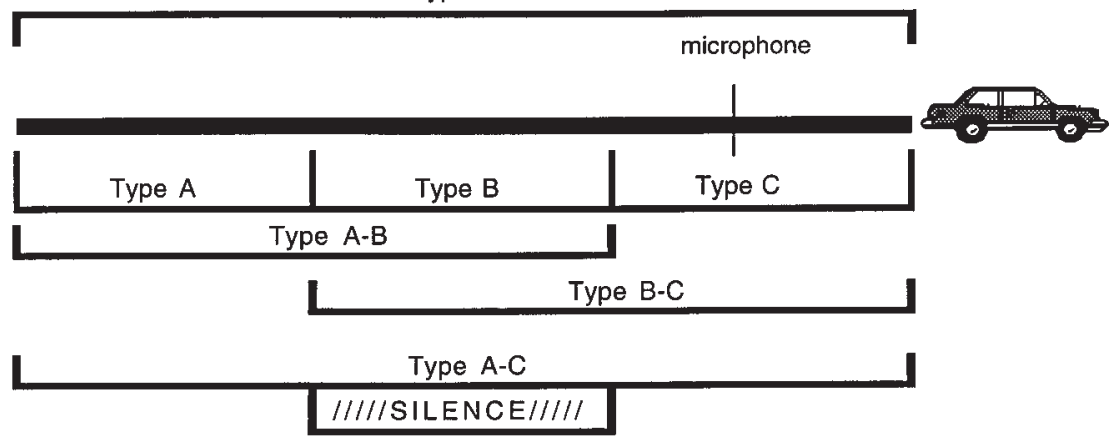

FIGURE 1 A pictorial representation of the signal types used (see text for details). 
The experimental group of main interest was Group NFN (no feedback Session 1, feedback Session 2, no feedback Session 3). This feedback schedule was similar to the critical group in the Jagacinski et al. (1983) study and provided the most direct test of the effects of feedback withdrawal. Groups NNN (no feedback, no feedback, no feedback) and NFF (no feedback, feedback, feedback) functioned primarily as experimental controls with which the performance of participants' in Group NFN could be compared. Group NNN allowed us to track participants' performance without feedback but with extended practicing of the task. By testing performance in Session 3 across Groups NFN and NNN, we could determine whether Group NNN performance was due to prior feedback exposure or simply nonfeedback-related practice.

Participants in Group NFF received no feedback during the first session but were given feedback in both the second and third sessions. Comparing the third session of Groups NFN and NFF provides an additional measure of whether feedback withdrawal caused performance to revert. In addition, there was some concern that because of the length of the experiment, participants could become tired of the task by the third session. If this were the case, and performance in the third session decreased even with feedback, this information would be important to accurately interpret the performance of participants in Group NFN during Session 3.

During each session, participants were seated in front of a computer. They were told that they would be listening to recordings of a car approaching them and that their task was to indicate when the car would reach them. The experimenter then demonstrated the experimental setup using illustrations. Participants were told to imagine that they were standing by the side of the road facing an approaching car, and they were to indicate with a key press at what point the car would have reached them. They were informed that some of the recordings were of the entire event, whereas others were partial recordings, but that they should respond to both in the same manner and simply indicate the point at which the car would have reached them had it continued on at a constant speed. Participants started each stimulus presentation by pressing a button and then indicated their judgment by pressing the button a second time. The second button press did not end the playback of the recording but simply registered the time of the listener's response. The computer was programmed to perform millisecond timing between key presses so the participant's time-to-arrival judgment was easily recorded. At this point, depending on Group assignment, some participants were given feedback, whereas others were not. After the feedback, there was a short delay, and the participant was then able to initiate the next recording.

The stimuli were delivered to participants in blocks, each containing all seven signal types of a single approach speed (15 or $25 \mathrm{mph}$ ). Blocking the trials in this way was necessary because of computer system constraints. Within each block, each signal type was presented five times, yielding 35 trials that were randomized within the block. Four blocks were presented during each experimental session, two for the 15-mph and two for 25-mph tokens. Counterbalancing was used for the 
block presentation, with presentation ordered such that half of the participants heard a block of 15 -mph tokens first $(15,25,15,25)$ and the other half received a 25 -mph block first $(25,15,25,15)$. In each session, participants received 10 trials of each of the 14 conditions, producing 140 total trials $(7$ signal types $\times 2$ speeds $\times$ 10 trials). In addition to the experimental trials, at the beginning of each session each participant received six practice trials to become accustomed to the stimuli and task. The six practice trials consisted of Condition Types A-B, A-C, and A-B-C from both speeds and included feedback only if listeners were in a feedback portion of the experiment. Each session lasted about $1 \mathrm{hr}$, and each person participated in three sessions on consecutive days.

\section{RESULTS}

The analyses were performed in an effort to address the issue of whether listeners retained any effects of learning on withdrawal of feedback. Before any calculations were performed, response times deviating greater than $3 \mathrm{sec}$ were removed to eliminate timed-out trials and biases due to attentional errors (approximately $2 \%$ of each participant's total trials). To evaluate listeners' performance across sessions, an absolute deviation score was computed for each judgment (Rosenblum et al., 1993). This deviation score was calculated by taking the absolute value of the difference between the actual time of passage and the judged time of passage. As in Rosenblum et al., the absolute deviation measure was used because signed deviation scores can mathematically cancel each other, yielding spuriously near-perfect mean scores. Furthermore, our interest was in the performance of groups across sessions when pooled over participants. Figure 2 shows the means for the absolute deviation scores averaged over participants for each session and group.

Although the absolute deviation scores are useful to portray pooled data, they do not capture the patterning of anticipatory or lag bias in participant judgments. For this reason, information regarding the proportion and magnitude of overestimation and underestimation across each session and group are presented in Figure 3.

Because our main interest was in investigating the effects of feedback withdrawal on performance, we performed an analysis of variance (ANOVA) on the data for NFN group listeners, using absolute deviation from correct as the dependent variable. This ANOVA tested the factors of Order of Presentation (15 or 25 $\mathrm{mph}$ first), Session (1, 2, or 3), Speed (15 or $25 \mathrm{mph}$ ), and Signal Type (A, B, C, $\mathrm{A}-\mathrm{B}, \mathrm{B}-\mathrm{C}, \mathrm{A}-\mathrm{C}$, and $\mathrm{A}-\mathrm{B}-\mathrm{C})$. The Geisser-Greenhouse epsilon was used to calculate probability values to correct for specific departures from homogeneity of variance and sphericity for each factor (Stevens, 1990). This ANOVA revealed that only the factors of Session and Type were significant, $F(2,18)=15.34, p<$ .001 , and $F(6,54)=9.63, p<.001$, respectively. In addition, there were reliable two-way interactions for Session $\times$ Type, $F(12,108)=3.25, p<.05$, and Speed $\times$ 


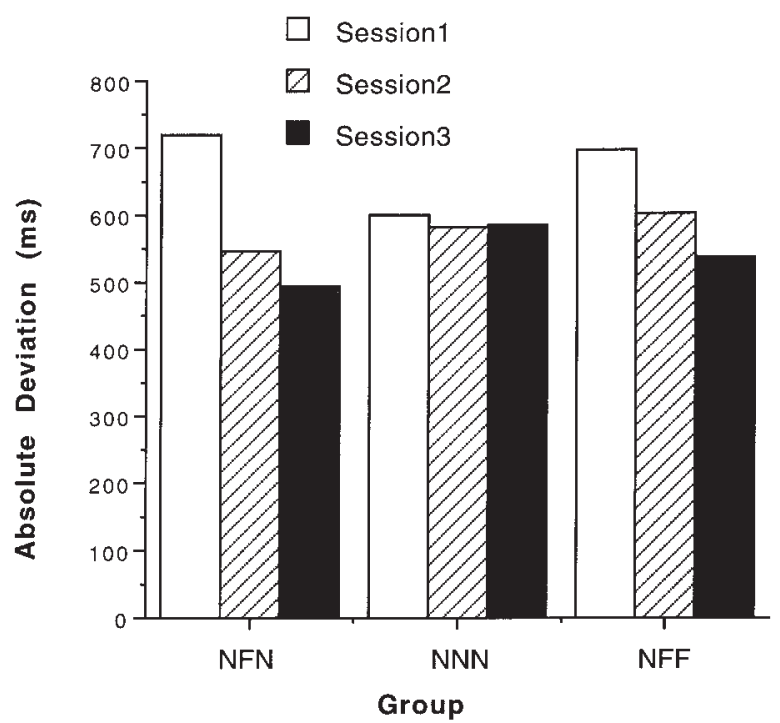

FIGURE 2 Absolute deviation scores for each session and participant group. NFN = no feedback Session 1, feedback Session 2, no feedback Session 3; NNN = no feedback Session 1, no feedback Session 2, no feedback Session 3; NFF = no feedback Session 1, feedback Session 2, feedback Session 3.

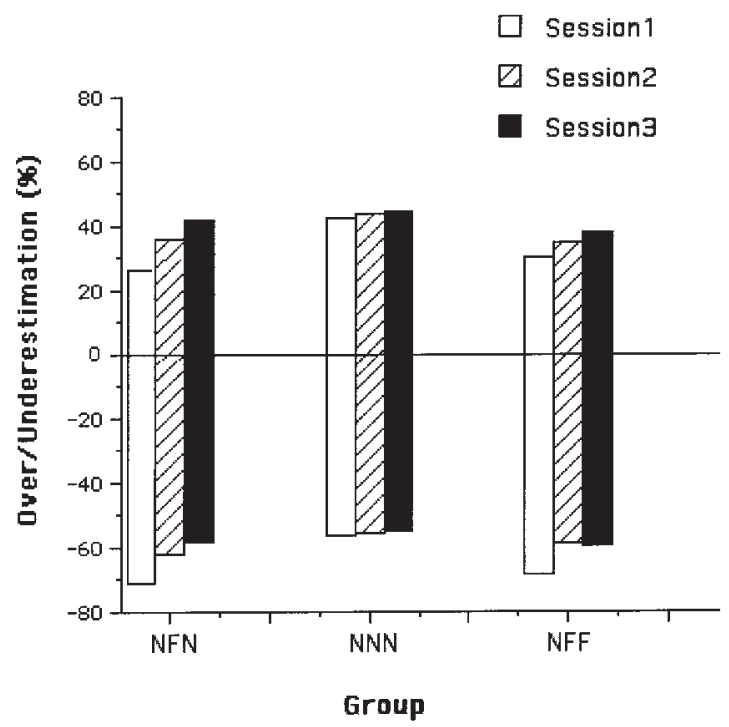

FIGURE 3 Percentage of overestimation and underestimation judgments for each session and each participant group. Positive scores indicate overestimations, and negative scores indicate underestimations. NFN = no feedback Session 1, feedback Session 2, no feedback Session 3; $\mathrm{NNN}=$ no feedback Session 1, no feedback Session 2, no feedback Session 3; NFF = no feedback Session 1, feedback Session 2, feedback Session 3. 
Type, $F(6,54)=6.63, p<.01$. There was also a three-way interaction of Session $\times$ Speed $\times$ Type, $F(12,108)=4.505, p<.01$.

Because several interactions reached significance, any interpretation of the effects for Session and Type should be made with caution; however, they can provide an overall view of the data. The effect for Type indicates that performance was dependent on which portion of the signal participants were judging. Figure 4 shows the average deviation scores for each signal type averaged over the NFN group participants.

Given the hypotheses driving this research, the Session and Session $\times$ Type effects for the NFN group were the most important to investigate further. The effect for Session demonstrates that performance improved during the experiment (Ms $=720,547$, and $494 \mathrm{msec}$ for Sessions 1, 2, and 3, respectively). Not surprisingly, the introduction of feedback produced a significant improvement in performance between Sessions 1 and 2, $F(1,18)=16.5, p=.002$. However, of primary importance, there was no significant difference in performance between Sessions 2 and 3 , $F(1,18)=1.55, p>.2$. This indicates that there was no reversion of performance on withdrawal of feedback.

The significant Session $\times$ Type interaction indicates that listeners' changing performance across sessions was dependent on which portion of the trajectory was heard. Figure 5 shows the average deviation scores for each signal type and each session averaged over the NFN group participants. Of principle interest was whether there were some signal types in which performance reverted or became

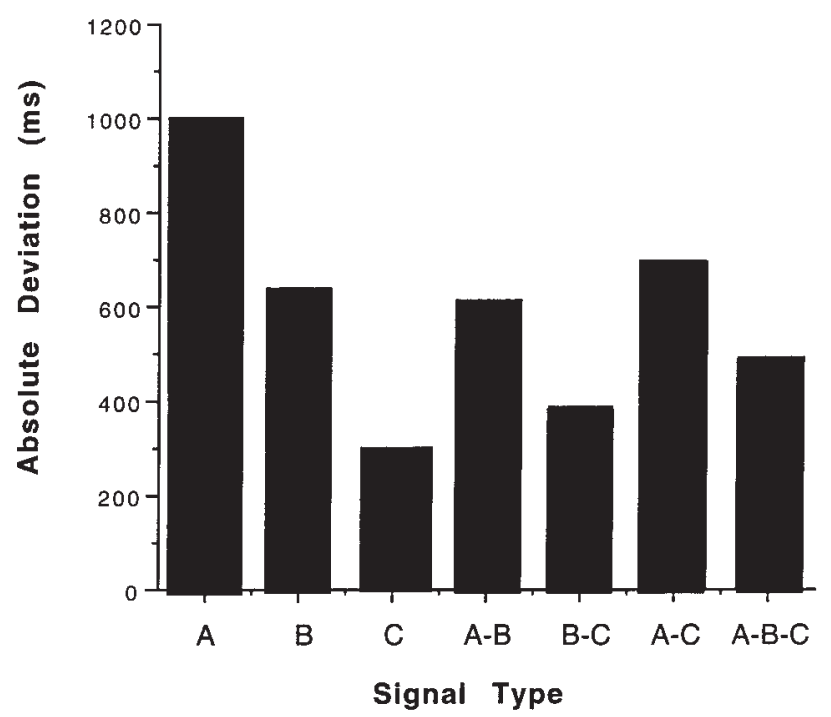

FIGURE 4 Absolute deviation scores for each signal type for participants in the NFN (no feedback Session 1, feedback Session 2, no feedback Session 3) group. 


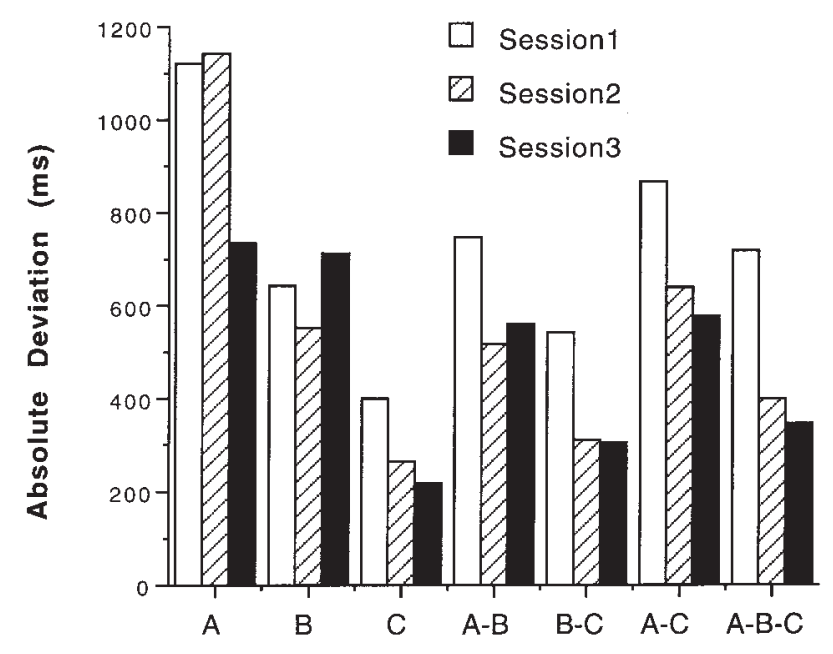

Signal Type

FIGURE 5 Absolute deviation scores for each signal type and session for participants in the NFN (no feedback Session 1, feedback Session 2, no feedback Session 3) group.

significantly worse. None of the Session $\times$ Type contrasts showed reversion of performance. Contrasts showed that the only type of signal that showed a significant reversion of performance between Sessions 2 and 3 was Signal Type B. This effect is interesting as it may help us understand exactly what is being learned in time-to-arrival judgments. There are several factors that could affect learning in this case, including signal duration and the specific portion of the trajectory involved. Clearly, further research should be performed looking at this issue more specifically.

These data indicate that judgment accuracy did not revert toward original performance after feedback was withdrawn. This suggests that listeners learned to improve their performance with feedback and that the benefits of this learning were maintained after feedback was withdrawn. Before drawing this conclusion however, another explanation must be addressed. It is still possible that the performance observed for the NFN group in Session 3 was simply a consequence of nonfeedback-related practice. To determine whether sustained learning through feedback accounted for Session 3 performance, it is essential to compare performance with the two other participant groups.

Performance of NNN and NFF group participants is represented in Figure 2. As can be seen by surveying the Session 1 means for each group, there is a problem in using the absolute deviation scores for these comparisons. Although each group received the same treatment during the Session 1, the average performance when pooled over participants, was different. The means for Session 1 were 720,601, and $697 \mathrm{msec}$ for the NFN, NNN, and NFF groups, respectively. 
Simply based on random assignment, listeners in the NNN group performed slightly better at the beginning of the experiment. These differential initial results pose a problem for assessing relative group performance through the absolute deviation measure. Accordingly, an alternate dependent measure was used to perform planned comparisons.

Each participant's absolute deviation score was converted into a relational measure such that his or her performance for each session was measured relative to his or her initial starting performance. To calculate this relational measure, the mean absolute deviation score was computed in each type and speed condition for Session 1 for each listener. These scores were considered to be the listener's initial starting performance. Each participant's mean absolute deviation score was then computed for each type and speed condition and divided by the initial starting performance for the specified speed and type condition. This relational measure reflects subsequent performance relative to initial performance. Therefore, performance equal to initial performance (no improvement) would yield a value of 1.0. Figure 6 shows the means of this relative measure for each group and session.

Converting the values into relational scores had little effect on the overall pattern of the NFN data. Session and Type effects were significant, $F(2,18)=3.89, p$ $<.05$, and $F(6,54)=2.15, p=.06$, respectively. The two-way interactions for

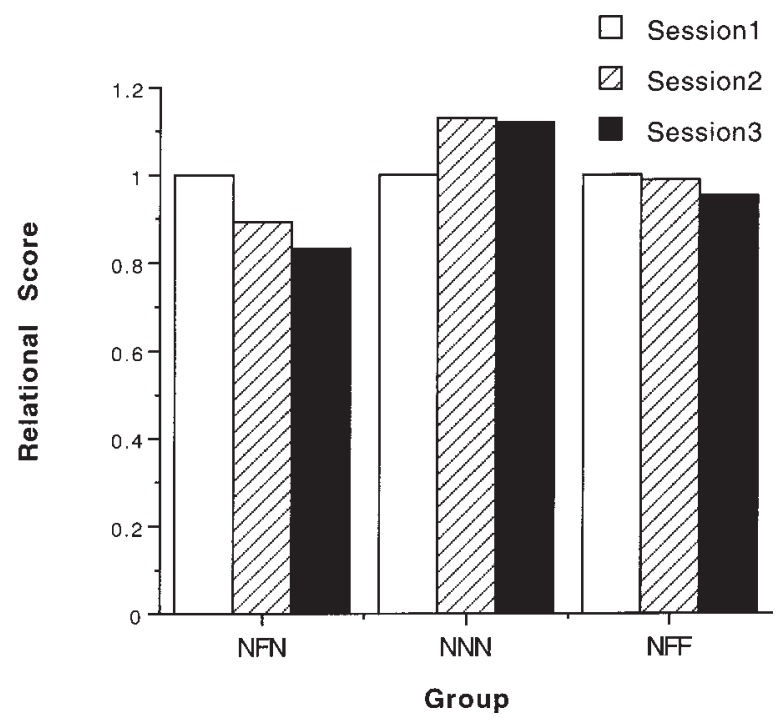

FIGURE 6 Scaled deviation scores for each session and participant group. NFN = no feedback Session 1, feedback Session 2, no feedback Session 3; NNN = no feedback Session 1, no feedback Session 2, no feedback Session 3; NFF = no feedback Session 1, feedback Session 2, feedback Session 3. 
Session $\times$ Type, $F(12,108)=3.13, p<.05$, and Speed $\times$ Type, $F(6,54)=5.868$, $p<.01$, and the three-way interaction of Session $\times$ Speed $\times$ Type, $F(12,108)=$ $5.209, p<.05$, were unaffected by the relational conversion.

As with the absolute deviation scores, the Session and Session $\times$ Type effects for the NFN group are the most important. The effect for Session demonstrates that performance improved during the experiment $(\mathrm{Ms}=1.0,0.89$, and 0.83 for Sessions 1, 2, and 3, respectively). Under closer inspection, marginal improvement was found between Sessions 1 and 2, $F(1,18)=3.1, p=.09$, whereas no difference was found between Sessions 2 and $3, F(1,18)=1.0, p>.3$. As with the absolute deviation scores, these results did show improvement of performance with feedback that did not revert after feedback had been withdrawn. It should be noted that although the pattern of effects revealed in the absolute deviation scores did not change with the relational conversion, the magnitude of those effects tended to be slightly reduced. This is not surprising given the nature of the relational score transformation.

Planned comparisons were made to determine whether performance of participants in the NFN group in Session 3 was based on retention of learning through feedback. These comparisons tested whether performance in Session 3 for NFN group was statistically different than Session 3 performance in NNN and NFF groups. Performance in Session 3 for Group NFN did not differ significantly from Group NFF but did marginally differ from Group NNN, $F(1,18)=3.43, p=.08$. These results indicate that listeners who were initially given feedback that was subsequently withdrawn did not revert back to a performance level one would expect based only on practice effects (NFN vs. NNN group comparison). In fact, performance for the group from which feedback was withdrawn did not differ significantly from the NFF group that continued to receive feedback throughout Session 3 (NFN vs. NFF group comparison).

In summary, these data support the conclusion that performance feedback significantly improves anticipatory judgments accuracy and that this improvement is generally retained (for at least 1 day) after feedback is withdrawn.

\section{DISCUSSION}

The purpose of this experiment was to determine how feedback and withdrawal of feedback affects perception of auditory looming and, further, to ascertain whether a more natural stimulus context might allow performance feedback to induce sustained improvements. Our results clearly show that for at least the 1-day duration between our second and third sessions, the improvements were generally sustained after feedback was withdrawn. These results contrast with those of Jagacinski et al. (1983), who found that feedback withdrawal caused participants to revert to previous performance accuracy. This is noteworthy because Jagacinski et al. used roughly the same amount of feedback across the same amount of time as was used in this ex- 
periment. Interestingly, our results are more in accord with anecdotal evidence (e.g., baseball batting, DeLucia \& Cochran, 1985; catching, Whiting et al., 1970; auditory looming perception of the blind, Schiff \& Oldak, 1990) that implies that improvements in looming judgments through performance feedback are longer lasting.

Why might our study have produced such different results from those of Jagacinski et al. (1983)? There are several differences between the studies that could be responsible for the divergent learning effects. First, and most obviously, the Jagacinski et al. experiment used visual stimuli, whereas this study used auditory stimuli. It is possible that the two perceptual systems instantiate learning differently. However, it is unclear what benefit would come from such a difference, and a cognitive system whose learning strategies were dependent on modality would not seem parsimonious.

A second difference between the studies is the direction of the looming trajectories used for the stimuli. Jagacinski et al. (1983) tested trajectories that were transverse relative to the observer (moving perpendicular to the line of sight). This experiment tested trajectories that were directed nearly radial relative to listeners (closely passing by the point of observation). A number of studies report that observers are more accurate in judging transverse than radial trajectories (Kebeck \& Landwehr, 1988; Schiff, 1988; Schiff \& Oldak, 1990). In testing radial trajectories then, this study did not provide the easiest stimuli to judge. Thus, although it is the case that the trajectory type differed between experiments, it is not clear how this could account for the differential learning effects.

A third difference between Jagacinski et al. (1983) and this study lies in the occluded periods tested. There is a good deal of evidence from both auditory and visual looming literatures that the smaller the occluded period, the more accurate participants are in their judgments (Rosenblum et al., 1993; and see Schiff \& Oldak, 1990, for a review). This study included occluded periods as long as $4 \mathrm{sec}$, whereas Jagacinski et al. tested occlusion times only as long as $2.5 \mathrm{sec}$. Thus, it is also unclear how these methodological differences could account for the different learning effects.

Another factor distinguishing the two studies is the form of feedback provided. Jagacinski et al. (1983) provided feedback in the form of explicit time in seconds, whereas this experiment used graphical feedback. It is possible that access to these different types of feedback differentially influence the learning process. However, we believe that this is unlikely based on previous pilot work in our lab that showed no significant difference in auditory looming task performance using both types of feedback.

Instead, we argue that the most salient difference between the two experiments lies in the nature of the stimuli. The unnaturalness of the stimuli used by Jagacinski et al. (1983) could have contributed to their feedback withdrawal effects. As stated, their synthetic stimuli were not embedded in any environmental context, such that there was no natural law-based relation between the event and the infor- 
mation provided. From the ecological approach, it is this naturally lawful relation that allows information to directly specify meaningful environmental facts to an observer (E. J. Gibson, 1969; J. J. Gibson, 1979/1986; Turvey, Shaw, Reed, \& Mace, 1981). This experiment used natural auditory stimuli whose structure was related to the looming event it specified. Potentially then, this aspect of the stimuli allowed for an attunement to the appropriate invariants that was maintained after feedback was withdrawn. An experiment that implements Jagacinski et al.'s general paradigm (e.g., a visual stimulus moving transversely, with verbal feedback provided) but uses more naturally constrained stimuli would provide a direct test of this hypothesis.

\section{Implications for an Ecological Approach}

In showing that a more natural, law-based stimulus context supports sustained looming judgment improvements, these results are supportive of the ecological view of time-to-arrival perception. Potentially, these results might also be useful for addressing two critiques of the ecological approach to looming perception. Next, each of these critiques will be presented along with how they have been addressed in the literature and how these data bear on them.

The critique most directly addressed by our results concerns the evidence for reversion to prefeedback performance observed by Jagacinski et al. (1983). The ecological approach maintains that perceivers use higher order information, or invariants, available in the stimulation to judge impending contact or arrival (e.g., Jenison, 1997; Lee, 1976, 1992; Rosenblum, 1993; Shaw, McGowan, \& Turvey, 1991). From this approach, information is directly available to the perceiver to inform him or her about the time to arrival of a source. This information is prospective in nature: Information is available in earlier portions of the event to specify the future time of arrival of the source. From this perspective, learning occurs through the differentiation of already available information (E. J. Gibson, 1969; E. J. Gibson \& Spelke, 1983; Michaels \& Carello, 1981). Through experience or training, the listener learns to educate his or her attention to the relevant informational invariants. Thus, explicit performance feedback might serve to attune perceivers to the salient prospective information. It is not clear from the ecological perspective, however, why withdrawing feedback would cause perceivers to revert to prefeedback performance as occurred in the Jagacinski et al. study. After all, once feedback helps the perceiver detect the salient invariants, there is no obvious reason why feedback withdrawal should undercut this ability.

The ecological approach contrasts with a cognitive approach to looming perception in which perceptual learning occurs through a fine tuning of model parameters (e.g., Jagacinski et al., 1983). (It should be mentioned that E. J. Gibson, 1969, offered a similar discussion of general perceptual learning approaches making the distinction between embellishment, or cognitive, and differentiation, or ecological, theories.) According to the cognitive approach, providing performance feedback 
would allow for more accurate estimates of the parameters that enable better extrapolation judgment. Withdrawal of feedback, in turn, can induce at least one of the parameter values to shift back to its initial value. As stated earlier, it is this explanation that Jagacinski et al. offered to rationalize the reversion of performance observed in their study.

Thus, in showing performance reversion on feedback withdrawal, the Jagacinski et al. (1983) results were supportive of a cognitive approach to looming perception and potentially damaging to the ecological view. In contrast, our results, in showing no reversion of performance, are supportive of the ecological approach. As stated previously, a natural stimulus context, captured in this experiment and characteristic of most everyday looming events, could provide the requisite invariants to allow performance feedback to support long-term attunement.

The second general criticism of the ecological approach to looming perception concerns the effects of occluded period on judgment accuracy. According to ecological theory, the rate of change information from any part of the event, whether early or late in the trajectory, should be equally able to support time-to-arrival judgments. However, and as stated previously, there is substantial evidence that observers are more accurate when judging looming stimuli with shorter occluded periods. This observation is evident in both visual (Carel, 1961; McLeod \& Ross, 1983; Schiff \& Detwiler, 1979; Schiff \& Oldak, 1990; Todd, 1981) and auditory (Schiff \& Oldak, 1990; Rosenblum et al., 1993) domains. Accordingly, the ecological approach has been criticized in this regard (Schiff \& Oldak, 1990). However, proponents of the ecological approach have offered several reasons why these effects could be occurring (Carello \& Turvey, 1991; Cutting, 1986; Schiff \& Oldak, 1990). For example, the ecological approach maintains that even if sufficient specificational information is available, it may not be detected or attended to by the perceiver (e.g., Michaels \& Carello, 1981). Observers have relatively little experience with occluded trajectory stimuli, and it may be necessary to learn to attend to the available invariants that specify time to arrival.

Our results bear on this issue in showing that feedback does improve performance and that this improved performance can be maintained. As argued previously, the fact that accuracy does not revert on feedback withdrawal provides evidence that improved performance is a result of an education of attention to the salient invariants in our occluded stimuli. Our results revealed a sustained improvement in all but one of the occluded periods tested. Thus, these findings might alleviate some concern about the occlusion duration effects in showing that the effects could simply be a function of observers' relative inexperience with occluded stimuli and its salient information.

\section{What Might Be Learned?}

The question arises about what exactly our listeners learned to display sustained judgment improvements. Potentially, and as suggested previously, our listeners 
might have learned a general attunement to acoustic time-to-arrival information, for at least, approaching automobile sounds. For example, the learning might have involved an enhanced attention to the lawful change in acoustic intensity endemic to an approaching sound source signal (Jenison, 1997; Shaw et al., 1991). Likewise, our listeners might have improved attention to lawfully related spectral, Doppler-induced pitch, or relative reflectance changes in the signal (see Rosenblum, 1993).

However, the fact that our stimuli were comprised of only two recorded events (15- and 25-mph approach trajectories) necessarily limits the conclusions that can be drawn about the nature of the learning. ${ }^{1}$ It could be that our participants used feedback to attune to idiosyncratic characteristics of these particular stimuli. Our naturally recorded events might have included spurious sounds (e.g., gravel hitting the car's fender) that participants used as time markers or "acoustic landmarks" to improve their accuracy. These acoustic landmarks might have been used explicitly or subliminally by participants to calibrate their learning through feedback. Such landmarks were not obvious to our listeners or ourselves or on our visual inspection of signal spectrograms (displayed in Rosenblum et al., 1993). In fact, it was our impression that there was an imposed uniformity in the timbral dimensions of the stimuli due to the low-pass filtering conducted on the signals $(5 \mathrm{kHz})$. Still, it could be that subtle landmarks were used tacitly by our listeners, and the observed learning effects could be specific to the exact stimuli used in our experiments. (This is also a potential problem for the learning effects observed in Rosenblum et al., 1993).

One way to examine whether performance improvements are from learning a stimulus-specific or more general looming information property would be to test the transfer of training to novel auditory looming stimuli (e.g., of different speeds, durations, sound sources). Although the purpose of this study was to determine whether feedback-induced learning could be sustained after feedback withdrawal, future experiments that examine learning in object approach perception (auditory or visual) would benefit from testing transfer of training to novel stimuli.

It is our belief that with the appropriate stimulus context, transfer of training could be empirically demonstrated for auditory looming perception. This belief is based on a number of considerations. First, we believe that the improvements observed with our stimuli occurred through listeners learning general auditory looming informational parameters. As stated, we were not aware of any spurious acoustic landmarks in either of our original stimuli, either through auditory or visual inspection (of spectrograms). Listeners would have to remember a number of landmarks for each event, as well as one for most segments. (Recall that listeners show sustained improvement for all but one condition.) Furthermore, although it is possible that retention of stimulus-specific properties might occur across the hour of a single experi-

\footnotetext{
${ }^{1}$ We are grateful to reviewers Sverker Runeson and John Pittenger for making us aware of this issue.
} 
mental session, it seems less likely that these signal subtleties would be retained across the 24-hr period separating Sessions 2 (initial feedback) and 3.

Third, many real-world examples of sustained looming perception improvements would seem to involve transfer of training from situations in which feedback was available to those in which it is not. The aforementioned example of blind listeners performing more accurately in auditory looming experiments (Schiff \& Oldak, 1990) likely involves transfer of training. As stated, one way blind individuals can improve this skill is through performance feedback provided either verbally from a mobility instructor or from less-threatening auditory approach experiences (e.g., hugging a speaking loved one). In that Schiff and Oldak's experiment involved stimuli that were novel to the listeners and did not involve performance feedback, the heightened performance of blind participants was likely transferred from a general learning of acoustic time-to-arrival parameters. Clearly, other performance improvements in both auditory and visual time-to-arrival instances are based on a transfer of general informational attunement. Research in our laboratory is currently being conducted to examine this question.

Assuming that our results are not specific to our stimuli and a more general perceptual learning does occur, there are some practical implications of these findings. For instance, mobility trainers often implement feedback in teaching visually impaired listeners to successfully interact with their environments. Similarly, learning through feedback has proven to be an important component in using virtual acoustic displays for telerobotics and other technologies. In both of these examples, learning to use auditory time-to-arrival information would seem critical. Accordingly, mobility trainers and designers of virtual reality systems should be encouraged by our results. Our experiment demonstrated that training through feedback will improve performance and that this performance can be retained. Further research should be conducted to determine how long this retention in performance might last. The results of this experiment also suggest that retention of improved performance might be dependent on the use of stimuli that are related to the specified event through natural law. If so, it would be advisable for both mobility trainers and virtual reality engineers to implement either natural auditory displays or synthetic displays derived from natural constraints. It could very well be that it is this aspect of the information that supports attunement to invariants supporting longer term learning.

\section{ACKNOWLEDGMENTS}

This research was supported by an Intramural Grant from the University of California-Riverside.

We gratefully acknowledge the assistance of Krista Anderson, Kim Buttacalova, Fernando Cardenas, Jadon Davis, Bernie Galvez, Sharon Jain, John Mannerino, Heather McAfee, and Zan Sullivan for assistance in data collection and thank 
John Pittenger and Sverker Runeson for their reviews of an earlier version of this article.

\section{REFERENCES}

Brunswik, E. (1956). Perception and the representative design of psychological experiments (2nd ed.). Berkeley: University of California Press.

Carel, W. L. (1961). Visual factors in the contact analogue (Pub. No. R61ELC60). Ithaca, NY: General Electric Company Advanced Electronics Center.

Carello, C., \& Turvey, M. T. (1991). Ecological units of analysis and baseball's “illusions." In R. Hoffman \& D. Palermo (Eds.), Cognition and the symbolic processes: Applied and ecological perspectives (pp. 371-385). Hillsdale, NJ: Lawrence Erlbaum Associates, Inc.

Cutting J. E. (1986). Perception with an eye for motion. Cambridge, MA: MIT Press.

DeLucia, P. R., \& Cochran E. L. (1985). Perceptual information for batting can be extracted throughout a ball's trajectory. Perceptual and Motor Skills , 61, 143-150.

Flynn, S. B. (1994). The perception of relative mass in physical collisions. Ecological Psychology, 6, 185-204.

Gibson, E.J. (1969). Principles ofperceptuallearning and development. New York: Appleton-Century-Crofts.

Gibson, E. J., \& Spelke, E. S. (1983). The development of perception. In P. H. Mussen (Ed.), Handbook of child psychology (pp. 1-60). New York: Wiley.

Gibson, J. J. (1966). The senses considered as perceptual systems. Boston: Houghton Mifflin.

Gibson, J. J. (1986). The ecological approach to visual perception. Hillsdale, NJ: Lawrence Erlbaum Associates, Inc. (Original work published 1979)

Hellman, A. (1993). Auditory perception of impending collision: Effects of experimental paradigm and strategy in judged time-of-arrival. In A. Schick (Ed.), Contributions to psychological acoustics (pp. 323-336). Oldenburg, Germany: Bibliotheks.

Jagacinski, R. J., Johnson, W. W., \& Miller, R. A. (1983). Quantifying the cognitive trajectories of extrapolated movements. Journal of Experimental Psychology: Human Perception and Performance, 91, $43-57$.

Jenison, R. L. (1997). On acoustic information for motion. Ecological Psychology, 9, 131-152.

Kebeck, G., \& Landwehr, K. (1988). Optical magnification as event information. Unpublished manuscript. (Available from Klaus Landwehr, Hittorfstrasse 44, d-5300, Bonn, Federal Republic of Germany)

Lee, D. N. (1976). A theory of visual control of braking based on information about time-to-collision. Perception, 5, 437-459.

Lee, D. N. (1992). Common principle of guidance by echolocation and vision. Journal of Comparative Physiology, 171, 563-571

McLeod, R. W., \& Ross, H. E. (1983). Optic-flow and cognitive factors in time-to-collision estimates. Perception, 12, 417-423.

Michaels, C. F., \& Carello, C. (1981). Direct perception. Englewood Cliffs, NJ: Prentice Hall.

Rosenblum, L. D. (1993). Acoustical information for controlled collisions. In A. Schick (Ed.), Contributions to psychological acoustics (pp. 303-322). Oldenburg, Germany: Bibliotheks.

Rosenblum, L. D., Wuestefeld, A. P., \& Saldaña, H. M. (1993). Auditory looming perception: Influences on anticipatory judgments. Perception, 22, 1467-1482.

Runeson, S., \& Vedeler, D. (1993). The indispensability of precollision kinematics in the visual perception of relative mass. Perception $\mathcal{E}$ Psychophysics, 53, 617-632.

Schiff, W. (1988). Accuracy of judging time-to-contact in visual and audiovisual events: Event anisotropy. Unpublished manuscript. (Available from William Schiff, 1032 Shimkin Hall, New York University, New York, NY 10003)

Schiff, W., \& Detwiler, M. L. (1979). Information used in judging impending collision. Perception, 8, 647-658. 
Schiff, W., \& Oldak, R. (1990). Accuracy of judging time to arrival: Effects of modality trajectory and gender. Journal of Experimental Psychology: Human Perception and Performance, 16, 303-316.

Shaw, B. K., McGowan, R. S., \& Turvey, M. T. (1991). An acoustic variable specifying time-to-contact. Ecological Psychology, 3, 253-261.

Stevens, J. (1990). Intermediate statistics a modern approach. Hillsdale, NJ: Lawrence Erlbaum Associates, Inc.

Todd, J. T. (1981). Visual information about moving objects. Journal of Experimental Psychology: Human Perception and Performance, 7, 795-810.

Turvey, M. T., Shaw, R. E., Reed, E. S., \& Mace, W. M. (1981). Ecological laws of perceiving and acting: In reply to Fodor and Pylyshyn. Cognition, 9, 237-304.

Whiting, H. T., Gill, E. B., \& Stephenson, J. M. (1970). Critical time intervals for taking in flight information in a ball-catching task. Ergonomics, 13, 265-272. 